\title{
Characterization of the Hamamatsu R11265 multi-anode photomultiplier tube with single photon signals
}

\section{Luca CADAMURO, Marta CALVI, Andrea GIACHERO, Claudio GOTTI*, Matteo MAINO, Clara MATTEUZZI, Gianluigi PESSINA}

Dipartimento di Fisica, Univ. degli Studi Milano-Bicocca (IT)

and INFN, sezione di Milano Bicocca (IT)

In view of the upgrade of the LHCb RICH detectors, multi-anode photomultiplier tubes (MaPMTs) were chosen as the baseline devices. The most promising models were tested, the latest being the Hamamatsu R11265. This tube is arranged in a $8 \times 8$ matrix of $2.9 \times 2.9 \mathrm{~mm}^{2}$ pixels with a very small inactive border near the sides of the device. The total active area coverage is more than $75 \%$. The device is being tested for single photon response with a commercial LED as the source of single photons. Current-feedback operational amplifiers are used as fast charge sensitive amplifiers. The outputs of the operational amplifiers are acquired with a fast oscilloscope. The very high readout speed simulates the fast readout which is foreseen in the upgraded $\mathrm{LHCb}$, and provides excellent time resolution which helps in resolving the single photon events.

International Workshop on New Photon-detectors

June 13-15, 2012

LAL Orsay, France

* Speaker. 

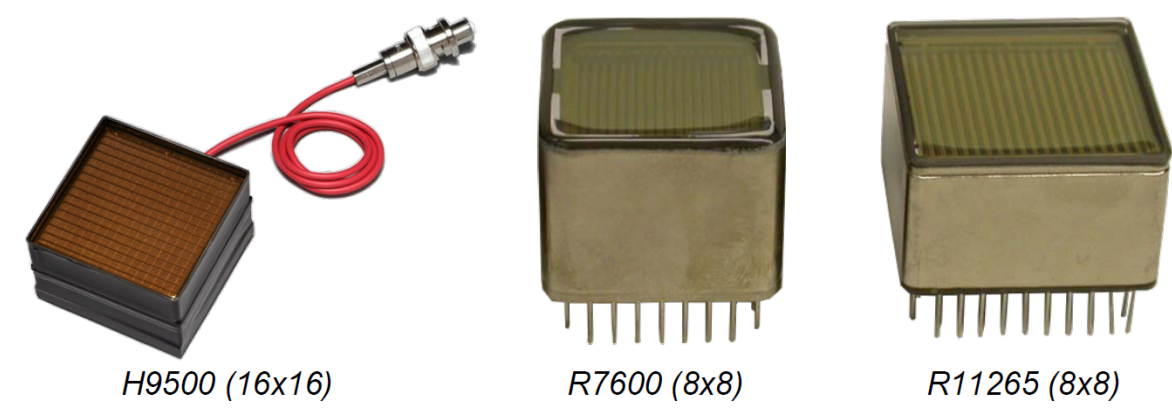

Figure 1: The Ma-PMTs characterized so far in Milano Bicocca in view of the LHCb upgrade: the H9500 (left), the R7600 (center) and now the R11265 (right), not to scale.

\section{Introduction}

Multi-anode photomultiplier tubes (Ma-PMTs) are pixelated photosensors with very low dark count rate, providing good sensitivity to single photons. Among the applications which can take advantage of these devices are Ring Imaging Cherenkov (RICH) detectors, used in high energy physics experiments to measure the velocity of charged particles, allowing their identification.

In view of the upgrade of the LHCb RICH detectors Ma-PMTs were chosen as the baseline devices $[1,2]$. The devices tested so far are shown in figure 1 . The H9500 was the first device tested. A non-negligible crosstalk between pixels was found at the single photoelectron level [3]. The Hamamatsu R7600 8×8 Ma-PMT was extensively studied and found compliant with the LHCb requirements, provided that adequate shielding to magnetic field was applied [4]. Due to the large inactive border near the sides the total area coverage of the R7600 is close to $50 \%$. The use of a lens would then be advisable to recover photodetection efficiency.

The R11265 Ma-PMT was recently introduced by Hamamatsu for use in cosmic ray detection experiments [5]. This device is similar to the R7600 but has a much smaller inactive border at the sides of the PMT, increasing the active area coverage to more than $75 \%$. This would allow the upgraded LHCb RICH to be designed without using lenses in front of the photosensitive plane, which would lead to a larger overall sensitivity of the detector. Even if the performance of the R11265 is expected to be close to that of the R7600 due to similarities in the design, the device must be characterized to verify its compliance with the LHCb RICH requirements in a LHCb-like environment.

\section{Measurement setup}

Figure 2 shows a scheme of the setup adopted to test the single photon response of the MaPMT at the single photon level. The blue LED is biased with a very small current, and part of the light it produces is coupled to a PMT pixel through an optical fiber. The PMT pixels are read out by commercial current feedback operational amplifiers (OPA695 by Texas Instruments) as described in [6]. The very wide bandwidth of the readout circuits allows a very fast shaping of the PMT pulses, which leads to a good separation of single photon events. The capability of the LED setup to generate and detect single photons was checked by comparing the spectrum obtained with the 


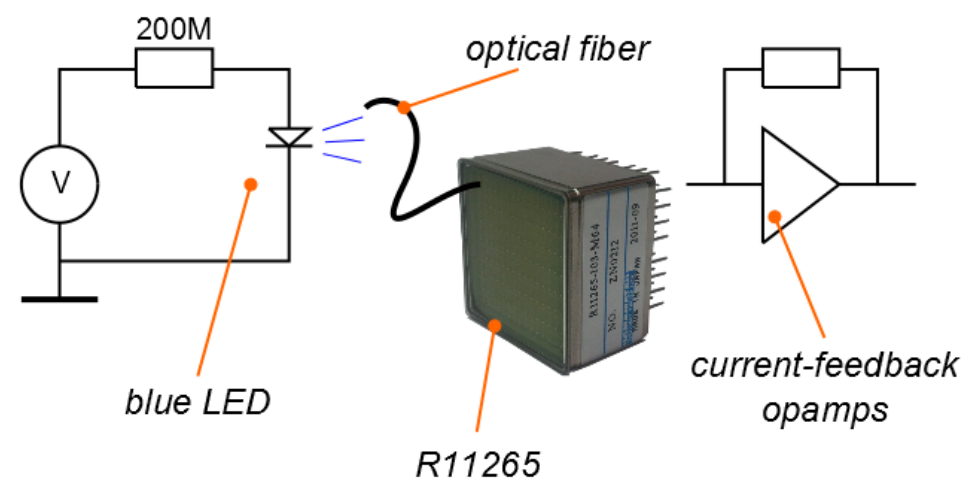

Figure 2: The setup used for the tests.

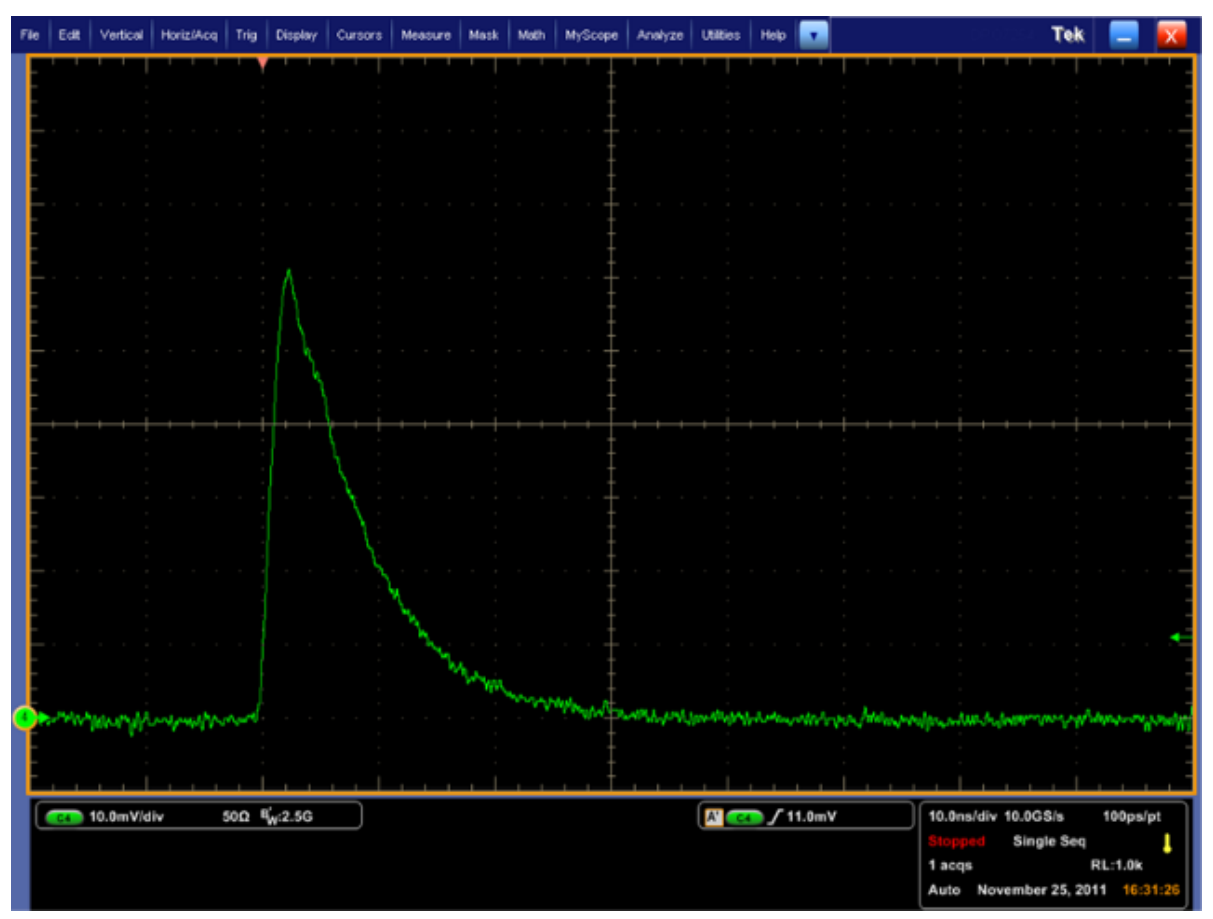

Figure 3: A typical single photon signal from the R11265, shaped by the current feedback operational amplifiers and acquired by the oscilloscope. The horizontal scale is $10 \mathrm{~ns} / \mathrm{div}$, the vertical scale is $10 \mathrm{mV} / \mathrm{div}$.

LED with the spectrum obtained using Cherenkov photons from a small crystal radiator and a radioactive source, as described in [4]. The perfect matching of the spectra confirms that the LED setup is capable to generate and detect single photon events. The operational amplifiers drive $50 \Omega$ terminated transmission lines to a Tektronix DPO7254 fast oscilloscope. Figure 3 shows the typical single photon signal as seen on the oscilloscope. The rise time is about $1 \mathrm{~ns}$, and the fall time is about $15 \mathrm{~ns}$, determined by the readout circuit. The charge collection time in this Ma-PMT is very small, of the order of $1 \mathrm{~ns}$, and no long tails or afterpulses are present. 


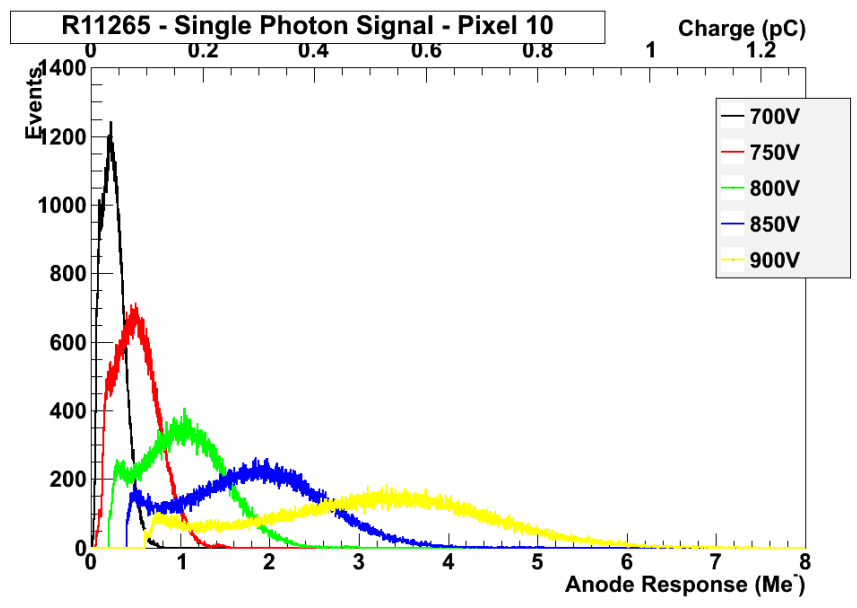

Figure 4: The spectra of a R11265 pixel for different values of the bias voltage.

\section{Bias, gain and uniformity}

The dependence of the gain of the device with applied bias voltage is shown in figure 4. With a bias voltage of $700 \mathrm{~V}$ the single photon peak sits at about 300 kiloelectrons (50 fC), reaching a value ten times larger if the high voltage is raised to $900 \mathrm{~V}$. The optimum bias voltage for use in the LHCb RICH depends on the choice of the readout electronics and on the expected event rate. With a low event rate, the gain can safely be increased to obtain a better signal to noise ratio. With a high event rate, a lower gain helps in minimizing the aging of the devices, but the readout electronics must then be able to efficiently trigger the smaller signals.

The uniformity table provided by Hamamatsu for the first R11265 we received is shown in figure 5, on the left side. The numbers represent the relative gain of the PMT pixels, the highest being 100. This measurement is performed with a continuous light source. The right side of figure 5 shows the comparison between single photon spectra of different pixels, obtained with the setup previously described. The measured spectra match the specifications, although obtained with single photon signals. The maximum gain spread within pixels of this device is 2.3. The readout electronics which will be adopted in the LHCb RICH must compensate this gain spread on a channel-by-channel basis, as commonly done with Ma-PMTs.

\section{Future plans}

The setup for single photon generation and readout was prepared using just a few commercial components and a fast oscilloscope. With this simple setup the R11265 Ma-PMT can be characterized for dark current and single photon response in a climatic chamber, to study the effects of temperature variation on gain and dark current rate. The tube will also be characterized for single photon response and crosstalk in a magnetic field up to $50 \mathrm{G}$ simulating the LHCb RICH conditions. Also other aspects such as aging and radiation hardness are planned to be studied. This characterization procedure will provide a full understanding of the electrical behaviour of the tube, which is of fundamental interest in order to validate the devices for use in the LHCb environment and to design the readout electronics for the upgraded LHCb RICH. 


\section{R11265 - Single Photon Signal at -850V}
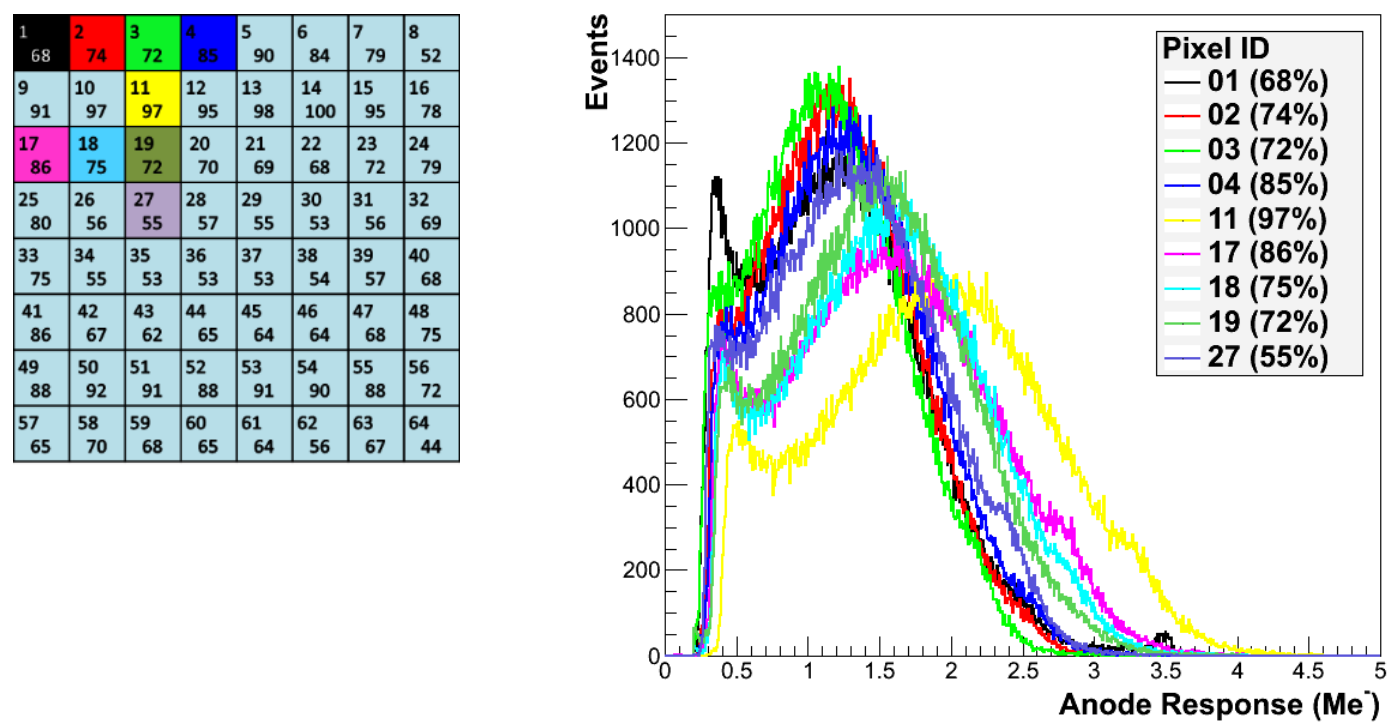

Figure 5: At the left side, the uniformity table provided by Hamamatsu with the first R11265 MaPMT we received. On the right side, the single photon spectra for some pixels highlighted with the corresponding colors.

\section{References}

[1] The LHCb Collaboration, Letter of Intent for the LHCb Upgrade, CERN-LHCC-2011-001; LHCC-I-018

[2] The LHCb Collaboration, Framework TDR for the LHCb Upgrade, CERN-LHCC-2012-007; LHCB-TDR-012

[3] C. Arnaboldi, et al., Crosstalk Study of the Single-Photon Response of a Flat-Panel PMT for the RICH Upgrade at LHCb, IEEE Transactions on Nuclear Science 57 (2010) 2267.

DOI: $10.1109 /$ TNS.2010.2052928

[4] M. Calvi, et al., Characterization of a Hamamatsu R7600 Multi-Anode Photomultiplier Tube with Single Photon Signals, Journal of Instrumentation 8 (2013) P02012.

DOI: 10.1088/1748-0221/8/02/P02012

[5] Y. Kawasaki, et al., The focal surface of the JEM-EUSO instrument, Astrophysics and Space Sciences Transactions 7 (2011) 167-169.

DOI: $10.5194 /$ astra-7-167-2011

[6] A. Giachero, et al., Current feedback operational amplifiers as fast charge sensitive preamplifiers for photomultiplier read out, Journal of Instrumentation 6 (2011) P05004.

DOI: 10.1088/1748-0221/6/05/P05004 